\title{
ANÁLISIS DE EDICIONES VESPERTINAS DE PRENSA PARA TABLETAS
}

Teresa Nozal, Ana González-Neira y Antonio Sanjuán

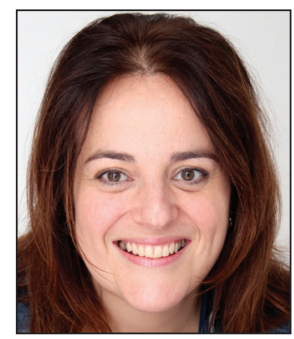

Teresa Nozal es profesora contratada doctora de periodismo en la Universidade da Coruña y vicedecana de la Facultad de Ciencias de la Comunicación. Doctora en periodismo por la Universidad de Navarra con una tesis sobre análisis de obras audiovisuales. Ha impartido docencia en las licenciaturas de periodismo y comunicación audiovisual. Coordina una línea de investigación sobre convergencia de medios periodísticos en tabletas del grupo Cultura y Comunicación Interactiva de la Universidade da Coruña. Tiene diversas publicaciones nacionales e internacionales sobre periodismo en dispositivos móviles.

http://orcid.org/0000-0002-2652-5898

tnozal@udc.es

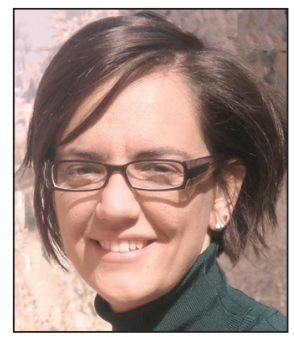

Ana González-Neira es profesora contratada doctora en la Facultad de Comunicación de la Universidade da Coruña (España). Es doctora en periodismo y licenciada en periodismo y ciencias políticas. Durante varios años fue profesora en la Università degli Studi di Genova (Italia) e investigadora invitada en la Universidad Nacional Autónoma de México. Es miembro del grupo de investigación Cultura y Comunicación Interactiva de la Universidade da Coruña. Ha publicado trabajos científicos sobre prensa en tabletas en revistas internacionales.

http://orcid.org/0000-0002-6369-0323

agonzalezn@udc.es

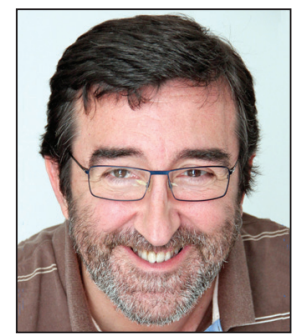

Antonio Sanjuán es profesor titular de comunicación audiovisual y publicidad en la Universidade da Coruña desde 2003. Doctor en ciencias de la información por la Universidad Complutense con una tesis sobre marketing de información documental electrónica. Trabajó como periodista y directivo en prensa escrita, radio y televisión durante más de 25 años. MBA en 1993. Es vicedecano en la Facultad de Ciencias de la Comunicación, de la que fue decano entre 2003 y 2009 . Pertenece al grupo de Cultura y Comunicación Interactiva. Ha publicado trabajos sobre estrategias y formatos comunicativos en tabletas.

http://orcid.org/0000-0001-7612-2838

sanjuan@udc.es

Universidade da Coruña Facultad de Ciencias de la Comunicación Campus de Elviña, 15071 A Coruña, España

\section{Resumen}

En un ecosistema mediático con productos periodísticos accesibles de manera instantánea, constante y ubicua, algunos diarios sorprenden con una edición vespertina para tableta incluso en países sin esa tradición en papel. Se plantean las claves para interpretar dicha aparente contradicción entre el ecosistema mediático actual y la estrategia que subyace en el lanzamiento de ediciones de la tarde en estos dispositivos móviles. Para ello se establece una muestra de aplicaciones nativas vespertinas de periódicos que no disponen de ella en su versión papel, se examinan y se entrevista a sus responsables. Entre las conclusiones destaca el peculiar papel que se asigna a estas ediciones dentro del proceso informativo diario pues más que un fin en sí mismas resultan ser un medio para mantener las tendencias de consumo previas.

\section{Palabras clave}

Tabletas, Periodismo, Periodismo digital, Medios, Periódicos, Multimedia, Convergencia, Aplicaciones.

\section{Title: Analysis of newspapers' evening editions for tablets}

\begin{abstract}
In a media ecosystem with news products available instantly, anywhere and at any time, some newspapers have sprung a surprise by publishing an evening edition for tablets, even in countries with no newsprint tradition. This article considers the keys to interpreting this apparent contradiction between the current media ecosystem and the strategy underlying the
\end{abstract}


launch of late editions for these mobile devices. To do so, we establish a sample of native evening newspaper applications not available in print, examine them and interview the prime movers. The conclusions reveal the unique role played by this newspaper type within the daily information process. More than just an end in themselves, they have become a medium that maintains previous patterns of consumption.

\section{Keywords}

Tablets, Journalism, Online journalism, Media, Newspapers, Multimedia, Convergence, Applications.

Nozal, Teresa; González-Neira, Ana; Sanjuán, Antonio (2014). “Análisis de ediciones vespertinas de prensa para tabletas". El profesional de la información, julio-agosto, v. 23, n. 4, pp. 393-400.

http://dx.doi.org/10.3145/epi.2014.jul.07

\section{Introducción}

En un contexto de crisis económica y de un ecosistema mediático líquido -es decir, con posibilidad de informarse instantánea, constante, ubicuamente y de modo personalizado- (Aguado-Terrón; Feijóo-González; Martínez-Martínez, 2013; Scolari, 2013), las empresas periodísticas han visto en la tableta una alternativa para abrir otros nichos de mercado (Cea-Esteruelas, 2013). En las primeras fases de mediamorfosis -transformación de un medio de comunicación como resultado de la combinación de cambios culturales y de nuevas tecnologías- (Fidler, 1998), en las que el papel se trasponía a las tabletas a través del pdf enriquecido y a la web a través de las webapp, empezaron a penetrar las primeras aplicaciones nativas con diseño y contenidos específicos para este soporte ${ }^{1}$. Algunas no importan de la web el sistema de actualización constante sino que imitan el de ediciones horarias del papel hasta el extremo de que se diseñan aplicaciones vespertinas en periódicos que no tienen esa edición en papel. Esta aparente contradicción responde a estrategias en las que los medios, el periodista y el receptor fluctúan entre el dato informativo instantáneo y el contenido con un tratamiento profundo asociado tradicionalmente al periodismo de calidad.

\section{El diseño y estructura de la mayoría de} aplicaciones vespertinas es más cercano a un suplemento de fin de semana que al de prensa matutina diaria e incluso dominical

Los estudios sobre tabletas revelan que se emplean mayoritariamente hacia al anochecer, cuando se terminan las jornadas laborales y se inicia el descanso (Santoro, 2013). Esto puede servir para justificar que algunos periódicos que en papel no tienen edición vespertina, incluso en países donde no hay tal tradición, hayan optado por incorporar a este dispositivo una versión nativa vespertina. En el contexto actual de crisis económica y periodística resulta un motivo insuficiente para justificar la inversión que exige, porque el dato se refiere al uso del dispositivo y no al consumo de aplicaciones periodísticas. Este trabajo pretende exponer la función que dichas aplicaciones nativas vespertinas cumplen en la estrategia de los periódicos y dar razón de su sentido en el entorno actual.

\section{Revisión bibliográfica}

La literatura científica sobre dispositivos móviles y prensa se ha abordado principalmente desde la perspectiva de la convergencia. En España la mayor parte de las aportaciones sobre prensa y tabletas se han recogido en obras sobre comunicación móvil (Scolari; Aguado-Terrón; Feijóo-González, 2012; Aguado-Terrón; Feijóo-González; MartínezMartínez, 2013; Carvajal, 2012), sin adentrarse en las propiedades y posibilidades específicas de este dispositivo frente a los smartphones. De similar enfoque es el artículo de Westlund (2013) sobre la producción de noticias en el periodismo móvil.

Limitando el análisis a las aportaciones centradas en tabletas exclusivamente, hay que tener en cuenta las contribuciones del Grupo de Pesquisa em Jornalismo Online de la Universidade Federal da Bahia en Brasil. Desde esta universidad se han realizado análisis descriptivos sobre la tactilidad (Cunha; Palacios, 2012), y el proceso de convergencia del que nacen algunas aplicaciones nativas (Barbosa et al., 2013; Barbosa; Da-Silva; Nogueira, 2013). Estos dos últimos estudios abordan casos concretos de aplicaciones vespertinas brasileñas para tabletas que recogen algunas de la muestra de este artículo. También cabe citar los trabajos de Canavilhas y Satuf (2013) así como el de Fernandes (2014), quien se centra exclusivamente en los contenidos audiovisuales de este tipo de aplicaciones. Hay que añadir el trabajo de Barsotti y Aguiar (2013) en el que se establece una comparación entre las propiedades formales del periodismo online definidas en otros textos y las que presentan las ediciones en tableta, marcando las potencialidades de este último dispositivo.

La bibliografía sobre tabletas se completa con el estudio de usabilidad, multimedia e interactividad en aplicaciones de prensa de Nozal y González-Neira (2013), el trabajo de Alonso-del-Barrio (2013) sobre mecanismos de participación, o los análisis formales de Parmeggiani (2013) y Suárez-Carballo y Martín (2013). Asimismo, cabe destacar las monografías sobre tableta y prensa de García (2012), que se centra en el diseño de aplicaciones periodísticas, y Valentini (2012) sobre las oportunidades de este soporte y los cambios de lenguaje y consumo que supone.

La mayor parte de estas investigaciones proponen conclusiones a partir del análisis descriptivo formal de las aplicaciones periodísticas en sus primeros años. Ninguna pretende dar sentido al surgimiento de ediciones temporales, 
como es el caso de las vespertinas, dentro del ciclo informativo diario en un contexto de información continua y ubicua.

\section{Muestra y método}

Se define un universo formado por diarios de España, donde surge esta investigación, y de países que pertenecen a un modelo mediático similar, de modo que el trabajo sea coherente y dé pie a futuros trabajos comparativos entre modelos. Dentro del paradigma pluralista polarizado (HaIlin; Mancini, 2008), además de España, se toman 3 países de Europa y 3 de Iberoamérica ${ }^{2}$. En Europa: Portugal, Italia y Grecia por estar en una coyuntura socioeconómica similar a España. En Iberoamérica: México, Brasil y Argentina por ser los tres estados con mayor PIB.

Una vez definidos los países que forman parte del universo, se identifican aplicaciones vespertinas, nativas y ligadas a un periódico que no disponga de dicha edición vespertina en papel que son el objeto de estudio de este trabajo. Tras la pertinente revisión queda en evidencia que Portugal, Grecia, Argentina y México no tienen ninguna edición que cumpla los requisitos. Se obtiene así una muestra compuesta por las siguientes aplicaciones, de las que se indica a continuación la fecha de aparición:

- Diário do Nordeste plus, de Diário do Nordeste, Brasil, 18 de enero de 2013;

- El mundo de la tarde, de El mundo, España, 29 de octubre de 2013. Dado que El mundo no dispone de edición para Android durante el período investigado, se limita la muestra a las aplicaciones para el sistema iOS.

- Estadão noite, de O Estado de S. Paulo, Brasil, 24 de abril de 2012;

- La repubblica sera, de La repubblica, Italia, 7 de noviembre de 2011;

- O globo a mais, de O globo, Brasil, 30 de enero de 2012.

Se combinan dos métodos en la investigación:

1. Un análisis de contenido de la muestra basado en datos cuantitativos extraídos tras aplicar tres fichas:

- En la primera se analiza si el texto de las ediciones vespertinas repite o complementa el de las matutinas del mismo día o si es exclusivo. Para ello se hizo una recogida de datos sobre el contenido de todas las noticias objeto de la muestra asignando un valor numérico (1) en cada caso. Dentro del contenido exclusivo se distinguió entre ligado a la agenda del día y atemporal. A partir de la suma de cada categoría se hallaron los porcentajes.

- La segunda ficha compara la nomenclatura de las secciones de la edición vespertina frente a la matutina para establecer si predominan secciones calientes o frías (es decir, más o menos vinculadas a la agenda del día). Para ello se hizo un vaciado del nombre de las secciones de cada aplicación de la muestra en cada uno de los dos días $y$, teniendo en cuenta su contenido, se asignó un valor numérico (1) en cada caso. A partir de la suma se calcularon los porcentajes.

- La tercera extrae datos sobre multimedia (vídeos, fotografías, infografías, audios, ilustraciones): tipo, cantidad e incorporación o no de galerías de fotos y vídeos. Se hizo un vaciado de contenidos estableciendo número de vídeos

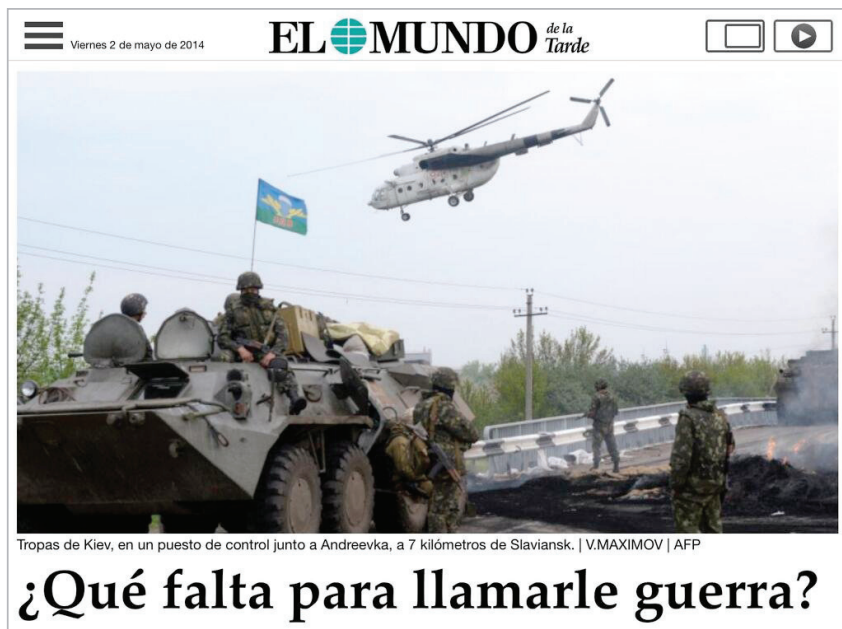

"Muchos muertos" en una dura ofensiva gubernamental tras sufrir dos bajas y perder tres helicópteros en el este | Los rebeldes se hacen fuertes en Slaviansk

- Al menos un muerto en una batalla campal en las calles de Odessa entre fieles a Kiev y afectos a Moscú | Ucrania reinstaura el servicio militar obligatorio - Cronología de un mes que desgarró Ucrania en dos mitades enfrentadas

ESPAÑA| DESAFIO SOBERANISTA
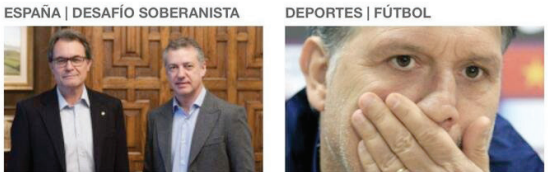

FIN DE SEMANA | CULTURA

Mas y Urkullu, juntos en el independentismo

Martino defiende a Pep y éste se defiende solo

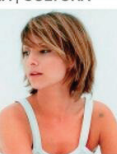

El mundo de la tarde

de busto parlante, vídeos de publicidad insertados en las noticias, otros que no responden a las categorías anteriores, fotos integradas en las noticias, infografías o gráficos, audios de publicidad, otros audios, y número de viñetas o ilustraciones. Además se determinó si cada aplicación objeto de la muestra integraba galería de fotos y de vídeos independiente.

Todas las fichas pasaron un proceso de testeo inter-codificadores llevado a cabo por los investigadores. La recogida de datos se efectuó del 11 al 15 de noviembre de 2013. Dichos datos se evaluaron para establecer las aparentes estrategias seguidas por los periódicos con sus ediciones vespertinas nativas.

2. Un método cualitativo a través de entrevistas semiestructuradas con los responsables de los periódicos para contrastar los datos obtenidos en el examen de la muestra. Estas entrevistas parten de cuestiones acerca de la estrategia de lanzamiento de la vespertina y de su consumo. Previo al vaciado de datos se identificó a los responsables de las ediciones para tableta. Posteriormente se realizaron las entrevistas durante diciembre de 2013 y se filtraron los resultados, contrastando los datos derivados de la observación de la muestra.

\section{Diseño y estructura: slow-information para infotabment}

El contexto en el que se prevé que serán consumidas las ediciones vespertinas influye en la elaboración de un producto específico. El carácter de soporte lean $b_{a c k}{ }^{3}$ de las tabletas (García, 2013) facilita la incorporación de piezas informativas en profundidad como reportajes, entrevistas o crónicas, frente a las noticias que responden exclusivamente a las tradicionales $5 \mathrm{w}$ (who, what, when, where, why). Los edito- 


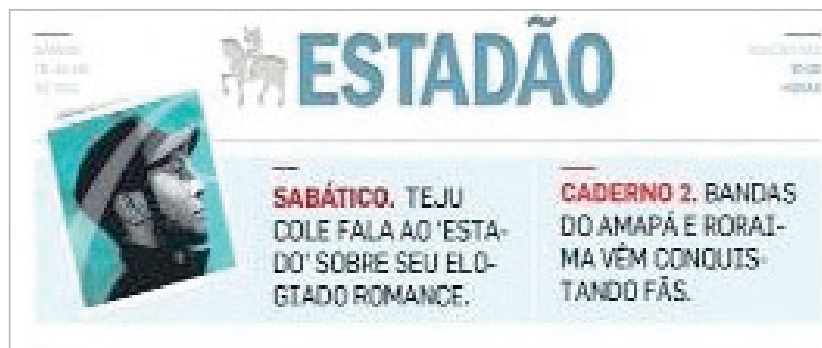

Necrows

Estado reselou que a milher de Sadok tere RS 18 miem contratos de obras

\section{Dilma afasta diretor executivo do Dnit após novas denúncias}

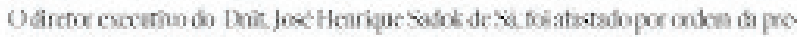

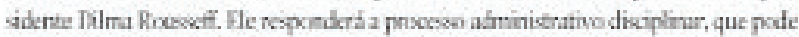

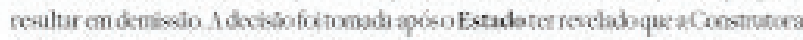

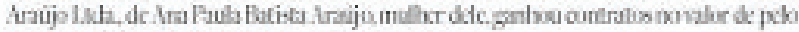
monk RS 18 milhiesparatciar donsemindwus.

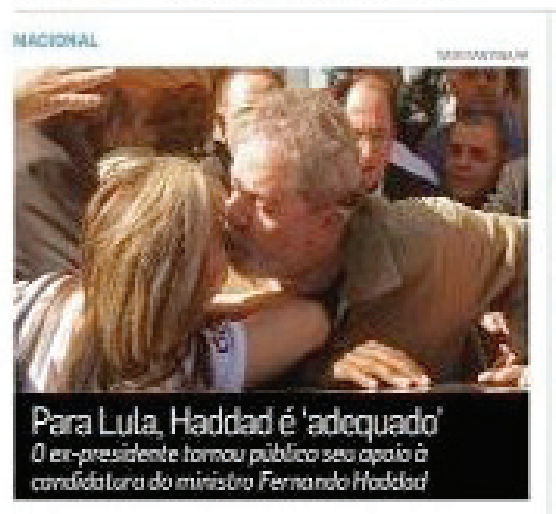

\section{tecovones \\ Sob pressilio, Obama dá ultimato ao Congresso Prossionalepelachirs epelasaptioins de caceitacasiode rispaco proikniculas Tstakis linidos, Barack tho. mo, dezaconyeseo praxide 36 horesparam

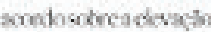

\section{FAAP A Faculdade do Futuro}

Estadão noite

res están produciendo para estas vespertinas formatos periodísticos de slow-information ${ }^{4}$ en los que los artículos en profundidad son los protagonistas. De este modo los heavy users $^{5}$ también pueden ver con interés estas ediciones.

En la conducta de infotabment no se busca información concreta sino que se pretende descansar navegando entre contenidos periodísticos de la tableta

Estos formatos orientados a slow-information se asocian con productos híbridos entre periodismo y entretenimiento que dan respuesta a la actitud de infotabment, adaptación del concepto infotainment (información + entretenimiento) a la conducta detectada en usuarios de tableta que no buscan información concreta sino que más bien pretenden descansar navegando entre los contenidos periodísticos que la tableta les brinda (Sanjuán; Nozal; González-Neira, 2013b). En las aplicaciones orientadas a infotabment, la actualidad de la agenda suele ser una parte, con cuestiones muy leídas al lado de otras de consumo no informativo (programas, ficción, cocina, salud, turismo, fiestas...). Este uso reposado se asocia con contenidos que basculan entre la actualidad tratada en profundidad y el entretenimiento. El diseño y estructura de la mayoría de aplicaciones vespertinas responde a este concepto de usuario, más cercano al lector de un suplemento de fin de semana que al de prensa matutina diaria e incluso dominical.

Todas las aplicaciones de la muestra excepto El mundo de la tarde y Estadão noite cuentan con más contenidos procedentes de secciones frías, entendidas como aquellas que no están marcadas exclusivamente por la agenda del día. En números globales el $60 \%$ corresponde a secciones frías frente al $40 \%$ de calientes, pero además las frías son considerablemente más extensas en todas las aplicaciones, salvo El mundo de la tarde. Sirven de ejemplo las recetas de $L a$ repubblica sera y de Diário do Nordeste plus, las críticas de libros, películas o discos de La repubblica sera y $O$ globo a mais (sección "Dicas a mais") y la columna sobre arquitectura y decoración de Diário do Nordeste plus. En Estadão noite, $O$ globo a mais y Diário do Nordeste plus las noticias de la agenda que se han producido desde el cierre de la edición matutina se concentran en una sección específica.

\section{Subyace una estrategia de fidelización del cliente en los diferentes momentos de la jornada: papel por la mañana, web durante el día y app vespertina al ano- checer}

Todas siguen la estructura de secciones frías, con la única excepción de El mundo de la tarde, y utilizan una maquetación arrevistada en la que se deja mucho espacio a la parte gráfica. Pedro Doria, editor ejecutivo de $O$ globo, ha confirmado que en el diseño de la vespertina se sigue la línea de las revistas Esquire o del grupo Condé Nast porque aportan más posibilidades de maquetación. Algo parecido realizan las demás vespertinas, exceptuando la de El mundo, con portadas con una imagen central que recuerda a la primera página de los suplementos dominicales. De este modo se alejan de los cánones de diseño de los periódicos matutinos.

Las versiones vespertinas no se editan durante el fin de semana. Entre los motivos expuestos por los responsables está la falta de personal, aunque probablemente también influya la necesidad de evitar duplicidades con otros productos como los suplementos dominicales. De hecho Juan Carlos Laviana, responsable de esta edición de El mundo, afirma que han comprobado que se produce un alto índice de descargas el fin de semana, por lo que han optado por "hacer un número de viernes con muchos temas de lectura intemporales, que se puedan leer el sábado o el domingo". Lo mismo confirma Fernanda Delmas, editora de $O$ globo a mais: "Mas a edição de sexta-feira sempre teve um caráter de edição de fim de semana, com mais conteúdo e mais soft news".

El mundo de la tarde es el diario más informativo de la muestra. Este apego a la agenda diaria que trasluce su estructura se corresponde con un diseño más sobrio y menos visual que las otras vespertinas. No obstante tras la experiencia de 


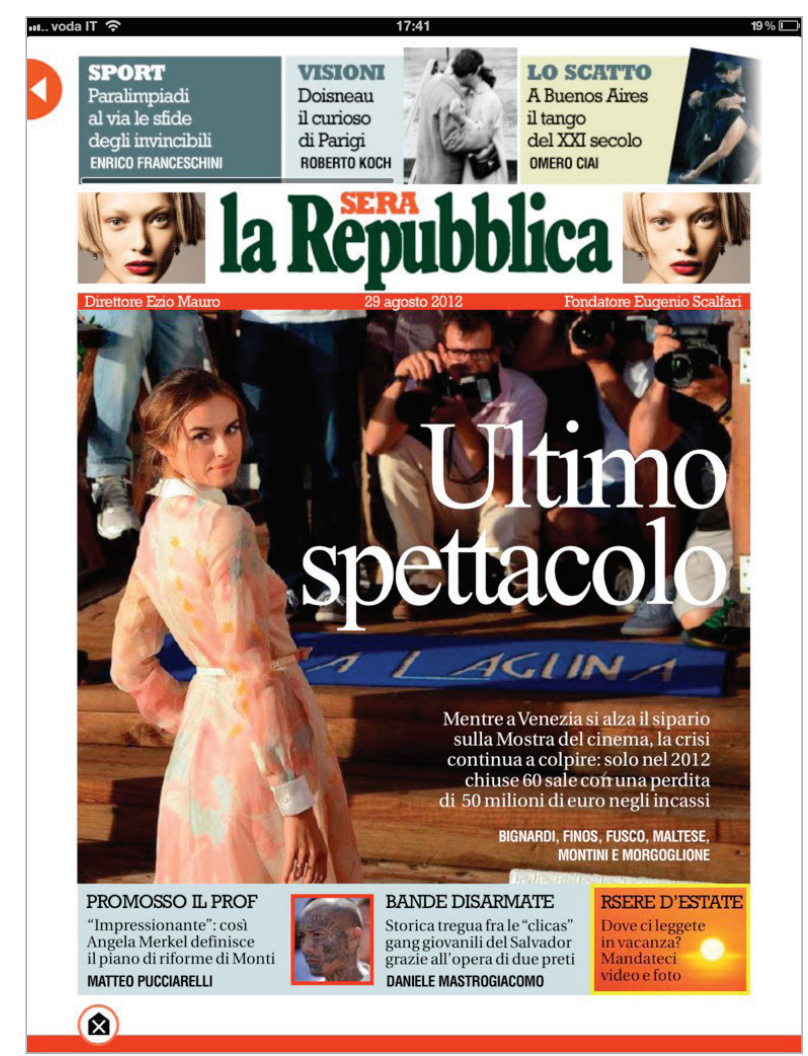

La repubblica sera

los primeros meses y ante la opinión de los lectores, se está restando espacio a los textos escritos y dando más peso a la parte gráfica, según confirmó su responsable, Laviana.

Las vespertinas se orientan a vender el producto estrella de la marca, que sigue siendo el papel o su equivalente en tableta: el pdf enriquecido

\section{Contenidos multimedia}

Las piezas multimedia se pueden diferenciar, en función de su localización en el periódico, entre situadas en galerías independientes e integradas en el texto. Todas las ediciones vespertinas incorporan galerías de fotografías pero ninguna de vídeos. En todos los casos las secciones de fotos del día están compuestas por imágenes muy llamativas visualmente y no tanto por las cuestiones más candentes de la agenda. Se favorece de este modo un consumo más cercano al entretenimiento, al estilo que practican los suplementos dominicales. Otra vez se detecta un producto orientado hacia una actitud de infotabment por parte del lector en el que lo periodístico se consume como distracción, ojeando vídeos como espectador pasivo de un programa y galerías de fotos ordenadas por criterios simples o sin ordenar.

En los contenidos multimedia integrados en el texto de la noticia, La repubblica sera es el periódico con mayor número de vídeos y fotos, el 33,57\% del total de los vídeos identificados en todas las aplicaciones y el $31,25 \%$ de las fotos, frente al Estadão noite en el extremo opuesto con el 4,65\% y el $9,38 \%$ de vídeos y fotos respectivamente.

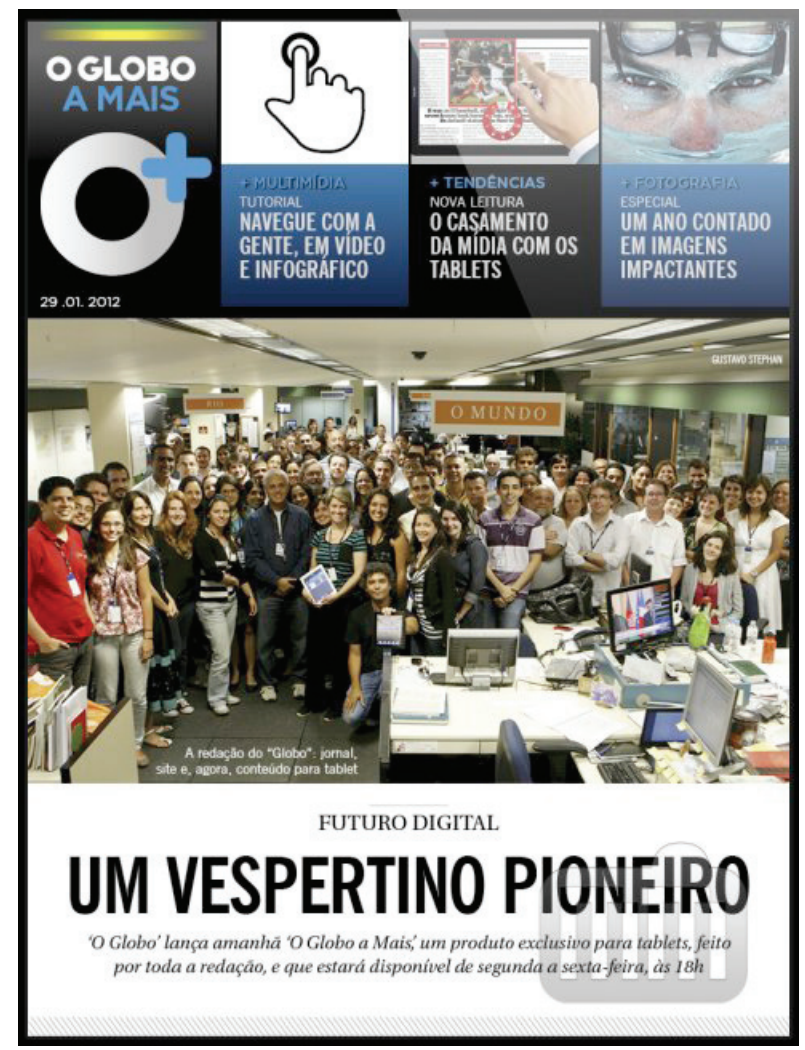

O globo a mais

En estos contenidos multimedia no siempre pesa tanto el motivo estético como ocurre en las galerías. Luca Fraioli, responsable de La repubblica sera, la aplicación con mayor número de vídeos y fotos, explica que al igual que los suplementos dominicales, publican reportajes de contexto dando peso a la parte audiovisual, opinión que también comparte Pedro Doria, editor ejecutivo de $O$ globo.

Gran parte de los multimedia de vídeo y audio proceden de otros medios del mismo grupo del periódico (RepubblicaTV, TV $D N$ y TV estadão) en un claro ejemplo de convergencia (Salaverría; García-Avilés; Masip-Masip, 2010) y aprovechamiento de sinergias. Los restantes provienen de agencia o son vídeos musicales y trailers. Asimismo cabe destacar en las apps vespertinas la presencia de contenidos ajenos al periodismo web como los audios de todas las ediciones, excepto $O$ globo a mais, que es la única que no los incorpora. La mayor parte de los vídeos y audios de producción propia introducen opiniones de expertos o columnistas sobre algunos sucesos del día, género poco frecuente en la parte multimedia de las webs de los periódicos (García-Orosa; López-García; Gallur-Santorum, 2013).

\section{Los contenidos basculan entre la actua- lidad tratada en profundidad y el entre- tenimiento}

El limitado empleo de infografías animadas que se ha detectado se puede explicar por la ausencia en las redacciones de prácticas consolidadas, como sí sucede en el caso del vídeo o el audio. La integración de códigos comunicativos procedentes de las animaciones queda como asignatura pendiente en estas aplicaciones para tableta. 


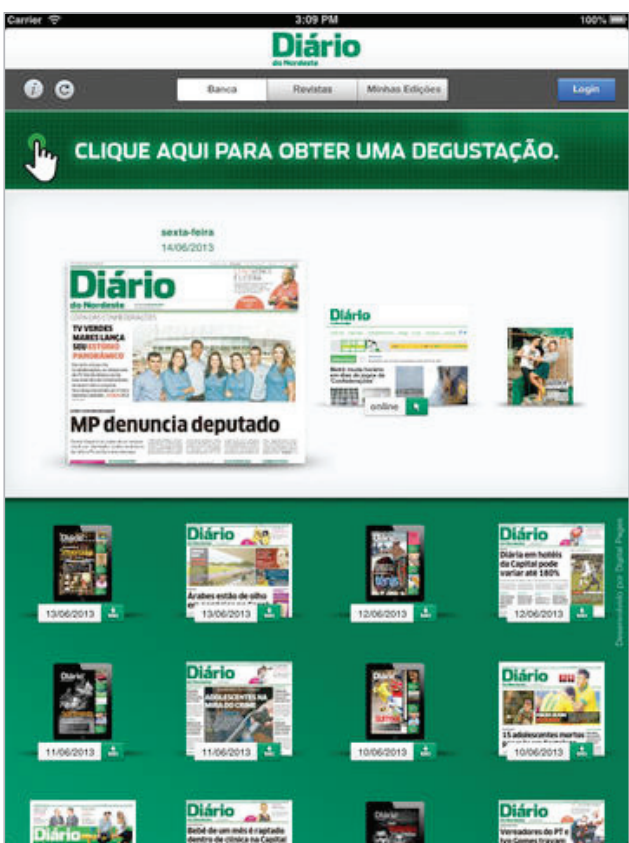

Diário do Nordeste plus

\section{Vespertino: aperitivo del matutino en la cadena informativa}

Estas ediciones suelen publicar noticias que no han sido introducidas previamente en la cabecera. El porcentaje de información exclusiva -entendida como aquella que ha sido elaborada específicamente para la aplicación vespertina- es del $82 \%$ en El mundo de la tarde, el $83 \%$ en La repubblica sera, en O globo a mais del 94\%, en Diário do Nordeste plus del $88 \%$ y en Estadão noite del 100\%. La mayoría de la información exclusiva relativa a la agenda del día es retomada en la edición matutina del día siguiente. De este modo las tabletas pueden convertirse en una vía más de negocio junto al papel y la web, fortaleciendo la estrategia multidispositivo, como confirman Doria (O globo) o Fraioli (La repubblica sera). Se diversifica la oferta para favorecer el pago de un paquete completo que abarque las tres vías y sea más atractivo. Según las declaraciones de responsables como Laviana, Fraioli o Delmas, la estrategia multiplataforma de los periódicos reside en lanzar productos diversos para momentos también diferentes.

Subyace una estrategia de fidelización del cliente al querer mantenerlo en los diferentes momentos del día: papel por la mañana, web durante el día y al anochecer app vespertina, que introduce noticias de la matutina del siguiente día, convirtiendo el ciclo informativo en un continuo. La vespertina se plantea como un medio de fidelización para el producto estrella, que sigue siendo el papel o su equivalente en tableta: el pdf enriquecido.

Por eso las ediciones vespertinas avanzan noticias exclusivas de la versión impresa del día siguiente. De hecho en Estadão noite se incorpora una sección denominada "Amanhã, no jornal O Estado de São Paulo", que adelanta parte de lo que publicará el pdf del día siguiente del mismo modo que "Panorama" en Diário do Nordeste plus. Por lo tanto aparentemente la estrategia de la empresa periodística sigue centrándose en vender el papel o el pdf del día siguiente más que una aplicación independiente y novedosa.

Los limitados datos de consumo facilitados por los responsables confirman esta situación. Por ejemplo en La repubblica sera el número de lectores corresponde sólo al $20 \%$ de los del pdf, en Estadão noite al $10-15 \%$ y en $O$ globo a mais se sitúa en la horquilla del $20-50 \%$. Por otro lado la lectura de estos productos conlleva un incremento del tiempo dedicado al consumo de contenidos. En el caso de $O$ globo se triplicaron las descargas y suscripciones. Según indica Pedro Doria: "Actualmente $O$ Globo tiene métricas de fidelidad impresionantes para su aplicación iPad, con un promedio de una hora y media por visita. Antes de añadir la edición de la tarde las visitas eran de sólo unos 26 minutos" (McAthy, 2013).

\section{Conclusiones}

Las ediciones vespertinas de los grupos periodísticos revisados han servido como taller de experimentación de dinámicas internas de producción y de narrativas multimedia. En todos los casos son la primera versión nativa que lanzan esas cabeceras por lo que aún no explotan todas las potencialidades que pueden proporcionar, como el mayor uso de las infografías y consiguiente enriquecimiento sensorial, o una mayor personalización e interactividad. Aun así constituyen un avance en la convergencia tecnológica, profesional y de contenidos, superan el hieratismo del pdf y resuelven el dilema de formular un producto diferente al sitio web y al impreso aprovechando las dinámicas de consumo de la tableta y la actitud de lean back.

La estrategia multiplataforma de los periódicos reside en lanzar productos diversos para momentos diferentes

Entre la muestra se distingue El mundo de la tarde por su diseño y por los aspectos tratados. De hecho las aplicaciones revisadas, exceptuando la de $E I$ mundo, no responden al perfil de ediciones vespertinas de un periódico convencional, ya que no prima la agenda del día. Más bien recuerdan a suplementos dominicales editados entre semana, tanto por tratar asuntos más atemporales como por el diseño arrevistado. Por consiguiente son publicaciones similares a las revistas digitales que incorporan un apartado para las cuestiones más relevantes del día, mientras que el resto corresponden a reportajes o piezas sobre materias frías (cine, literatura, decoración o música). Además dicho diseño, según declaran los responsables, multiplica las posibilidades multimedia. Constituyen en su mayoría productos orientados al infotabment.

Las cuestiones de actualidad que introducen estas aplicaciones son retomadas al día siguiente en la edición en papel o pdf enriquecido siguiendo una estrategia de fidelización del lector para orientarlo hacia la matutina. De este modo los grupos periodísticos siguen primando la edición en papel o pdf enriquecido de la mañana como primer objetivo de ventas y no tanto productos que supongan una rentabilidad por sí mismos. 
Las ediciones vespertinas refuerzan la conversión de las rutinas informativas en un proceso continuo con dos grandes paradas. Una a primera hora del día con el plato fuerte informativo. Otra al final del día, con noticias que han surgido tras el cierre de la matutina -como corresponde a una segunda edición- y que sirven como gancho para el periódico de la mañana siguiente, y que incorpora contenidos orientados a la actitud de infotabment más propios de suplementos dominicales. Entre ambas, la web funciona como un goteo continuo de datos informativos más que de contextualización y profundidad.

El mundo de la tarde se distingue de las demás vespertinas por su diseño y por los asuntos tratados, más similares a un periódico matutino convencional

En resumen la estrategia que subyace al fenómeno de las vespertinas es fidelizar al lector a la marca de la cabecera cuyo buque insignia sigue siendo el papel o pdf enriquecido matutino- reforzando la idea de "más productos y servicios por el mismo precio".

\section{Notas}

1. Para una clasificación de aplicaciones en tableta véase: Nozal y González-Neira (2013) y las posteriores, Suárez-Carballo y Martín (2013) y Sanjuán, Nozal y González-Neira (2013a) Respecto a la clasificación de contenidos en dispositivos móviles, no específicamente tabletas, Canavilhas y Satuf (2013) se basan en la realizada por Scolari, Aguado-Terrón y FeijóoGonzález (2012).

2. Siguiendo a Hallin y otros autores se considera que el sistema mediático de la mayoría de los estados iberoamericanos se incorpora por sus rasgos al modelo pluralista polarizado de los países del sur de Europa: Hallin y Papathanassopoulos (2002) y Figueras-Maz et al. (2012).

3. Habilidad para mantener la atención de los lectores en productos periodísticos durante períodos largos de tiempo frente a los dispositivos lean-forward como el smartphone.

4. El término slow-information nace de aplicar a los productos periodísticos el movimiento cultural slow surgido a partir del año 2000.

5. Heavy users. Adaptación del concepto homónimo de marketing al consumidor constante y en grandes cantidades de productos periodísticos, principalmente digitales.

\section{Bibliografía}

Aguado-Terrón, Juan; Feijóo-González, Claudio; MartínezMartínez, Inmaculada (2013). "Introducción de la cuarta pantalla al medio líquido". En: Aguado-Terrón, Juan; FeijóoGonzález, Claudio; Martínez-Martínez, Inmaculada. La comunicación móvil hacia un nuevo ecosistema digital. BarceIona: Gedisa, pp. 11-26. ISBN: 9788497847827

Alonso-del-Barrio, Estrella (2013). "Interactividad y participación en los medios adaptados para tabletas: las posibili- dades del periodismo 3.0". Estudios sobre el mensaje periodístico, v. 19, pp. 35-44.

http://revistas.ucm.es/index.php/ESMP/article/view/41959

Barbosa, Suzana; Da-Silva, Fernando; Nogueira, Leila (2013). "Análise da convergência de conteúdos em produtos jornalísticos com presença multiplataforma". Revista do programa de pós-graduação em mídia e cotidiano, Junho, v. 2, n. 2, pp. 139-162

http://www.ppgmidiaecotidiano.uff.br/ojs/index.php/ Midecot/article/view/52

Barbosa, Suzana; Da-Silva, Fernando; Nogueira, Leila; Almeida, Yuri (2013). "A atuação jornalística em plataformas móveis. Estudo sobre produtos autóctones e a mudança no estatuto do jornalista". Brazilian journalism research, v. 9, n. 2, pp. 1-20. http://bjr.sbpjor.org.br/bjr/article/view/549

Barsotti, Adriana; Aguiar, Leonel (2013). “Produção de notícias para dispositivos móveis: a lógica das sensações e o infotenimento". En: Canavilhas, João. Notícias e mobilidade: o jornalismo na era dos dispositivos móveis. Covilha: Universidade Beira Interior, pp. 295-318. ISBN: 9789896541026 http://www.livros/abcom.ubi.pt/book/94

Canavilhas, João; Satuf, Ivan (2013). "Jornalismo en transição: do papel para o tablet... ao final da tarde". En: Fidalgo, Antonio; Canavilhas, João (eds.). Comunicação digital. 10 anos de investigação. Coimbra: Minerva Coimbra, pp. 3560. ISBN: 9789727983421

http://www.researchgate.net/profile/Joao_Canavilhas/ publication/260190085_Jornalismo_em_transio_do_papel para_o_tablet_ao_final_da_tarde/file/9c96053009e225f570.pdf

Carvajal, Miguel (2012). "Estrategias de distribución del contenido periodístico en dispositivos móviles. Análisis comparativo de los principales editores de prensa española". En: Actas IV Congreso intl latina de comunicación social: Comunicación, control y resistencias, pp. 1-13. ISBN: 9788415698067 http://www.revistalatinacs.org/12SLCS/2012_actas/128_ Carvajal.pdf

Cea-Esteruelas, María-Nereida (2013). “Cybermedia economics: revenue model and sources o financing". El profesional de la información, julio-agosto, v. 22, n. 4, pp. 353-361. http://dx.doi.org/10.3145/epi.2013.jul.12

Cunha, Rodrigo; Palacios, Marcos (2012). "A tactilidade em dispositivos móveis: primeiras reflexões e ensaio de tipologias". Contemporanea, set.-dez., v. 10, n. 3, pp. 668-685.

http://www.portalseer.ufba.br/index.php/contemporaneaposcom/ article/viewFile/6575/4672

Fernandes, Juliana (2014). "Lo audiovisual en productos periodísticos exclusivos para tabletas: observando las tendencias en cuanto a sus formas y funciones". En: IV Congreso intl de la Asociación Española de Investigadores en Comunicación. AE-IC, pp. 202-305. ISBN: 9788469594346 http://www.aeic2014bilbao.org/download/aeic2014bilbao_ comunicaciones.pdf

Fidler, Robert (1998). Mediamorfosis: Comprender los nuevos medios. Buenos Aires: Granica. ISBN: 9506412618

Figueras-Maz, Mònica; Mauri-Ríos, Marcel; Alsius-Clavera, Salvador; Salgado-de-Dios, Francesc (2012). "La precarie- 
dad te hace dócil. Problemas que afectan a la profesión periodística". El profesional de la información, enero-febrero, v. 21, n. 1, pp. 70-75.

http://dx.doi.org/10.3145/epi.2012.ene.09

García, Mario R. (2012). The iPad design lab. Apple Store.

García, Mario R. (2013). “Extra! Extra! Lean back, lean forward. Read all about it". Garcia Media, 9 julio.

http://www.garciamedia.com/blog/articles/pextra_extra_ lean_back_lean_forward._read_all_about_it_p

García-Orosa, Berta; López-García, Xosé; Gallur-Santorum, Santiago (2013). "Análisis de la adaptación a la red en los editoriales on line de cinco países europeos". Revista latina de comunicación social, n. 68, pp. 485-501.

http://www.revistalatinacs.org/068/paper/986_usC/20_Orosa.html http://dx.doi.org/10.4185/RLCS-2013-986

González-Neira, Ana; Nozal, Teresa; Cunha, Rodrigo (2014). "Aplicaciones vespertinas de periódicos para tabletas: infoTabment y fidelización". En: Actas del IV Congreso intl Asociación Española de Investigación de la Comunicación, Bilbao, enero. ISBN: 9788469594346

http://www.aeic2014bilbao.org/download/aeic2014bilbao_ comunicaciones.pdf

Hallin, Daniel; Mancini, Paolo (2008). Sistemas mediáticos comparados. Tres modelos de relación entre los medios de comunicación y la política. Barcelona: Editorial Hacer. ISBN: 9788496913127

Hallin, Daniel; Papathanassopoulos, Stylianos (2002). "Political clientelism and the media: Southern Europe and Latin America in comparative perspective". Media, culture \& society, v. 24, n. 2, pp. 175-195.

http://dx.doi.org/10.1177/016344370202400202

McAthy, Rachel (2013). “Evening iPad editions: eight tablet publishing tips". Journalism.co.uk, July 5.

http://www.journalism.co.uk/news/evening-ipad-editionseight-tablet-publishing-tips/s2/a553468

Nozal, Teresa; González-Neira, Ana (2013). “La interactividad en las aplicaciones periodísticas para iPad italianas y españolas". Estudios sobre el mensaje periodístico, v. 18, pp. 639-648. http://revistas.ucm.es/index.php/ESMP/article/view/40943

Parmeggiani, Brenda (2013). “A convergência entre o jornal e os novos media: do impresso ao tablet - um estudo dos jornais portugueses pelo Laboratório de Pesquisa em Mobilidade e Convergência Midiática". En: III Congresso intl de ciberjornalismo (Cobciber). Observatório do Ciberjornalismo. ISBN: 9789899819900

http://cobciber3.files.wordpress.com/2013/03/livro-deatas-iii-cobciber-parmeggiani.pdf

Salaverría, Ramón; García-Avilés, José-Alberto; Masip-
Masip, Pere (2010). "Concepto de convergencia periodística". En: López, Xosé; Pereira, Xosé. Convergencia digital. Reconfiguración de los medios de comunicación en España. Santiago de Compostela: Universidad de Santiago de Compostela, pp. 41-64. ISBN: 9788498873795 http://dspace.si.unav.es/dspace/bitstream/10171/23730/1/ cap3_concepto_de_convergencia_periodistas_pp41-64.pdf

Sanjuán, Antonio; Nozal, Teresa; González-Neira, Ana (2013a). "Perception of journalistic contents in print and iPad version. Spanish case La voz de Galicia”. En: Rizvanog, Kerem. Research and design innovations for mobile user experience. Hershey: IGI Global, pp. 115-128. ISBN: 9781466644489

Sanjuán, Antonio; Nozal, Teresa; González-Neira, Ana (2013b). "Usabilidad, interactividad y contenidos multimedia en la prensa para iPad: el caso de El país, El mundo, $A B C$ y La razón". En: Canavilhas, João. Notícias e mobilidade: 0 jornalismo na era dos dispositivos móveis. Covilha: Universidade Beira Interior, pp. 73-98. ISBN: 9789896541026

Santoro, Pier-Luca (2013). "Il piacere laico del mattino". MediaHub, 18 aprile.

http://giornalaio.wordpress.com/2013/04/18/il-piacerelaico-del-mattino

Scolari, Carlos (2013). "De las tablillas a las tablets: evolución de las emagazines". El profesional de la información, enero-febrero, v. 22, n. 1, pp. 10-18.

http://es.scribd.com/doc/126345039/De-las-tablillas-a-lastablets-evolucion-de-las-emagazines http://dx.doi.org/10.3145/epi.2013.ene.02

Scolari, Carlos; Aguado-Terrón, Juan; Feijóo-González, Claudio (2012). "Mobile media: towards a definition and taxonomy of contents and application". International journal of interactive mobile technologies, v. 6, n. 2, pp. 29-38. http://online-journals.org/i-jim/article/view/1880 http://dx.doi.org/10.3991/ijim.v6i2.1880

Suárez-Carballo, Fernando; Martín, Juan-Ramón (2013). "Exploratory study of tablet publication models: visual communications and usability systems". Communication \& society / Comunicación y sociedad, v. 26, n. 3, pp. 49-66.

http://www.unav.es/fcom/comunicacionysociedad/es/ resumen.php?art_id $=458$

Valentini, Elena (2012). Dalle gazzette all'iPad. II giornalismo al tempo dei tablete. Milano: Mondadori. ISBN: 9788861842069

Westlund, Oscar (2013). "Mobile news. A review and model of journalism in an age of mobile media". Digital journalism, v. 1, n. 1, pp. 6-26.

http://www.tandfonline.com/doi/pdf/10.1080/21670811.2 012.740273

http://dx.doi.org/10.1080/21670811.2012.740273 